\title{
Empirical Modeling of Intra-BAN Ranging Errors Based on IR-UWB TOA Estimation
}

\author{
J. Hamie, B. Denis, R. D’Errico \\ CEA-Leti Minatec Campus \\ 17 rue des Martyrs, 38054 Cedex 09 \\ Grenoble, France \\ jihad.hamie@cea.fr
}

\author{
C. Richard \\ Univ. de Nice Sophia-Antipolis/UMR CNRS 6525 \\ Parc Valrose, 06108 Cedex 2 \\ Nice, France \\ cedric.richard@unice.fr
}

\begin{abstract}
In this paper we present a model accounting for dynamic intra- Wireless Body Area Network (WBAN) ranging errors based on Impulse Radio-Ultra Wideband (IR-UWB) Time Of Arrival (TOA) estimation in the $[3.1,5.1] \mathrm{GHz}$ and $[3.75$, $4.25 \mathrm{GHz}$ frequency bands. The latter is compliant with one mandatory band imposed by the IEEE 802.15.6 standardization group. Relying on time-variant on-body channel measurements, we draw our ranging error model as a mixture-based conditional probability density function, taking into account i) dynamic Signal to Noise Ratio (SNR) variations and ii) channel obstruction conditions, i.e. Line Of Sight (LOS) vs. Non Line Of Sight (NLOS), experienced over representative on-body links while walking. The density parameters evolution is thus characterized as a function of the previous channel and SNR configurations, illustrating e.g. missed/false path detection effects under low SNR. Ultimately this self-contained model could be incorporated within realistic simulations for motion capture and/or localization based on the IR-UWB WBAN technology.
\end{abstract}

\section{Categories and Subject Descriptors}

C.2.1 [Network Architecture and Design]: Wireless communication; D.3.3 [Systems and Technology]: System design issues, and performance modeling; H.1.2 [Models and Principles]: Miscellaneous

\section{General Terms}

Experimentation, Measurement, Performance

\section{Keywords}

Body Shadowing, IEEE 802.15.6, Impulse Radio, Non Line Of Sight, On-Body Propagation, Ranging Error, Time Of Arrival, Ultra Wideband, Wireless Body Area Network.

\section{INTRODUCTION}

Wireless Body Area Networks (WBANs) have recently gained significant research interest due to their utility in various application fields such as healthcare, security, sports, entertainment, human motion capture and navigation [1], [7], [8], [10]. In the WBAN context, the cooperative localization functionality consists in locating the nodes placed on a given body, relying on peer-to-peer range measurements. These measurements can be performed over on-body radio links (i.e. intra-WBAN ranging) and/or possibly between nodes that belong to different WBANs (i.e. inter-WBAN ranging). This new add-on is considered as an important enabling feature within future WBAN applications such as large-scale human motion capture and/or coordinated group navigation. In this context, the Impulse Radio - Ultra Wideband (IR-UWB) [6], [9] benefits from fine multipath resolution capabilities for precise range measurements based on Time Of Arrival (TOA) estimation. In the field of cooperative localization however, most of the algorithmic investigations carried out so far still consider unrealistic and synthetic TOA-based ranging errors under pedestrian mobility [1], [8], [10], hence biasing somehow the performance assessment in practical operating conditions. In particular, as far as we know, there does not exist any ranging-oriented parametric model that can really account for dynamic UWB intra-WBAN links.

In this paper, we consider characterizing and modeling such TOA-based ranging errors, using representative UWB onbody channel measurements, which were carried out under typical pedestrian walking [3]. More specifically, we take into account the dynamic link obstruction conditions experienced under body mobility, namely Line Of Sight (LOS) and Non Line Of Sight (NLOS) conditions alternatively. The variation of the resulting model parameters is also studied and discussed as a function of a controlled Signal to Noise Ratio (SNR) within synthetic received multipath signals. On this occasion, we illustrate false/missed first path detection phenomena under low SNR and NLOS conditions, as well as asymptotically ideal detections under higher SNR and LOS conditions.

This paper is structured as follows. In Section 2, we introduce the intra-WBAN TOA-based ranging principle. Section 3 then presents the methodology adopted to generate realistic TOA estimates out of channel measurements and subsequent synthetic channels with controlled $S N R$ values. In Section 4, we show the empirical probability density functions fitted to the resulting empirical errors, as well as the evolution of the related density parameters as a function of SNR and channel obstructions. Finally, Section 5 concludes 
the paper.

\section{INTRA-WBAN TOA-BASED RANGING 2.1 Single-Link On-Body Multipath Channel} IR-UWB is a radio technology that makes use of non sinusoidal impulses [5]. The main reasons motivating the use of IR-UWB in localization applications lies in its ability to provide high temporal resolution and accurate TOA estimation. Moreover, the IEEE 802.15.6 radio standard recently published for WBANs has promoted IR-UWB as a relevant physical layer in the very low power context [7]. Typically, distances between on-body sensor nodes can be determined out of the TOA information, provided that $n$-way ranging protocol transactions are also used [9]. Then the resulting distance estimates usually feed localization or tracking algorithms to position the mobile nodes [4]. Finally, for the $[3.1,5.1] \mathrm{GHz}$ and $[3.75,4.25] \mathrm{GHz}$ bands considered hereafter, it was previously shown in [3] that on-body channels suffer from significant shadowing, which is far dominating other distance-dependent effects. Accordingly, TOA estimation and its related error regimes are both expected to be strongly affected (and thus mostly conditioned) by dynamic body obstructions under mobility.

Over each intra-WBAN (i.e. on-body) link, the received signal can be typically represented as a function of the transmitted signal as follows:

$$
r(\tau)=\sum_{j=1}^{L_{p}} \alpha_{j} p\left(\tau-\tau_{j}\right)+n(\tau)=h(\tau) \otimes p(\tau)+n(\tau)
$$

where $h(\tau)=\sum_{j=1}^{L_{p}} \alpha_{j} \delta\left(\tau-\tau_{j}\right)$ is the multipath Channel Impulse Response (CIR) if $\delta($.$) is the Dirac delta function,$ $L_{p}$ is the number of multipath components, $\alpha_{j}$ and $\tau_{j}$ are respectively the amplitude and delay of the $j$-th multipath component, $p(\tau)$ is the transmitted pulse and $n(\tau)$ is an additive noise process.

Out of this observed signal, the TOA estimation step aims at determining the arrival time of the direct multipath component that would be ideally received in a free space propagation case. As revealed by equation (1), the TOA estimation quality depends on multiple factors such as the emitted pulse energy (and hence, the received pulse energy) in comparison with the noise floor, multipath fading effects (and hence, the occupied bandwidth), or signal obstructions. It is thus possible to generate false alarms due to early noisy realizations or to miss the direct path due to poor $S N R$ conditions and/or severe NLOS blockages. The latter tend to increase the apparent length of the direct path or they can even cause its absence, leading to overestimated ranges.

\subsection{Strongest Peak Detection through Matched Filtering}

Matched Filtering (MF), which is one of the most basic reception techniques, is generally characterized by relatively low complexity and low consumption, as claimed in [10]. Since WBAN nodes are strongly constrained with this respect, MF is also a rather convenient solution in our context. TOA estimates are thus obtained through strongest peak detection, by looking for the time shift that maximizes the cross-correlation function between the received signal and a local template, which theoretically corresponds to the unitary transmitted waveform, as follows:

$$
\begin{gathered}
c\left(\tau^{\prime}\right)=\int_{-\infty}^{+\infty} r(\tau) p\left(\tau-\tau^{\prime}\right) \mathrm{d} \tau \\
\hat{\tau}_{T O A}=\underset{\tau^{\prime} \in W}{\operatorname{argmax}}\left|c\left(\tau^{\prime}\right)\right|
\end{gathered}
$$

where $c\left(\tau^{\prime}\right)$ is the cross correlation function, and $\hat{\tau}_{T O A}$ is the estimated TOA in the temporal observation window $W$. The estimated distance is $\hat{d}=\hat{\tau}_{T O A} v$, where $v$ is the speed of light, assuming that the transmitter and the receiver are somehow synchronized, e.g. through Two Way Ranging protocol exchanges (i.e. assuming in first approximation that the Time Of Flight (TOF) is equivalent here to the TOA reading and that the errors affecting TOF measurements are restricted to that affecting TOA measurements). It will be seen in the following how to cope in part with the actual timing uncertainly when characterizing estimation errors out of real channel measurements.

Finally, the ranging error will be simply defined as the difference between the estimated TOA-based distance described previously and the actual distance, as follows:

$$
e=\hat{d}-d
$$

\section{RANGING ERROR MODELING METHO- DOLOGY}

This section describes the methodology adopted to draw our TOA-based ranging error model out of real channel measurements.

\subsection{Multipath Extraction out of Channel Mea- surements}

First of all, we consider the dynamic radio channels associated with the Hip-Chest and Hip-Wrist links from a past measurement campaign described in [3], where the total recording time was $4 \mathrm{sec}$ and consecutive temporal channel responses were collected every $20 \mathrm{~ms}$ in the band [3.1, 5.1] GHz. The measurements were performed under moderate human walk mobility in a typical indoor environment, resulting in a set of 200 time-stamped channel responses. For each response, multipath components were extracted using a CLEAN-like high resolution-algorithm [2] in [3.1, 5.1] GHz and $[3.75,4.25] \mathrm{GHz}$. A snapshot of the extracted CIR at the observation time-stamp $t_{n}$ can hence be expressed as:

$$
\hat{h}\left(t_{n}, \tau\right)=\sum_{j=1}^{\hat{L}_{p}\left(t_{n}\right)} \hat{\alpha}_{j}\left(t_{n}\right) \delta\left(\tau-\hat{\tau}_{j}\left(t_{n}\right)\right)
$$

where $\hat{h}\left(t_{n}, \tau\right)$ is the CIR extracted at the observation timestamp $t_{n}, \hat{L}_{p}\left(t_{n}\right)$ is the number of extracted multipath components, $\hat{\alpha}_{j}\left(t_{n}\right)$ and $\hat{\tau}_{j}\left(t_{n}\right)$ are respectively the amplitude and delay of the $j$-th extracted multipath component at time-stamp $t_{n}$.

Just like in [3], the dynamic power transfer function was also directly calculated out of the corresponding time-stamped frequency-domain measurements $H(t, f)$ in the band $B$ (anyway made available for RF calibration purposes), as follows: 


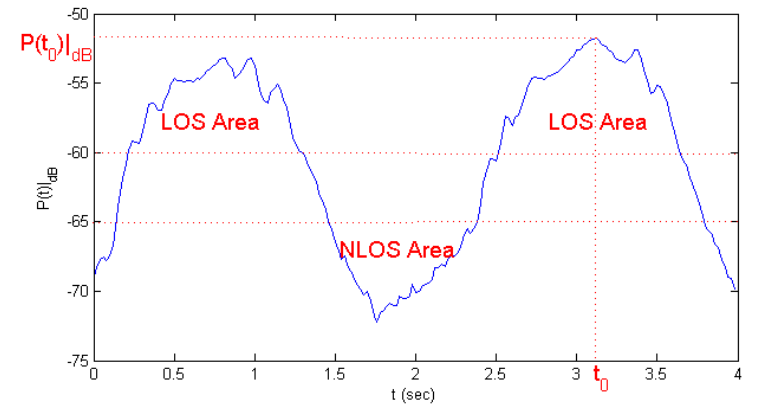

Figure 1: Dynamic variations of the power transfer function between the hip and the wrist under body mobility (standard walk), as a function of time $t$.

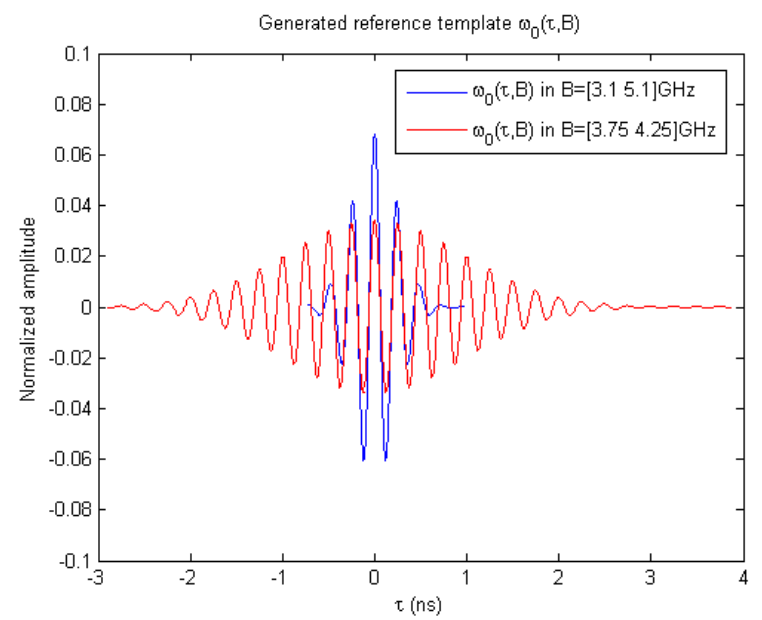

Figure 2: Energy-normalized templates $w_{0}(\tau, B)$ used for the generation of synthetic received signals and for correlation-based TOA estimation.

$$
P\left(t_{n}\right)=\frac{1}{b} \int_{B}\left|H\left(t_{n}, f\right)\right|^{2} \mathrm{~d} f
$$

where $b$ is the bandwidth of $B$, and $P\left(t_{n}\right)$ is the time-variant power transfer function, as illustrated on Figure 1 for the Hip-Wrist link.

As expected, this figure shows the strong body obstruction effects on the received signal attenuation. Typically NLOS channel conditions periodically lead to severe fades due to body shadowing under mobility.

\subsection{Generation of Synthetic Received Signals}

In order to synthesize a realistic received signal out of the extracted CIRs, as a function of a given initial SNR level and occupying a given bandwidth, a reference template waveform is required. Gaussian-windowed sine waves have thus been generated in the $[3.1,5.1] \mathrm{GHz}$ and $[3.75,4.25] \mathrm{GHz}$ bands, the latter being in compliance with one mandatory band specified by the IEEE 802.15 .6 bandplan. Figure 2 shows the corresponding reference templates normalized in energy. According to equation (1), those templates shall be convolved with the CIRs previously extracted out of real measurements, and an Additive White Gaussian Noise (AWGN) process with a two-sided power spectral density $N_{0}$ (i.e. $\left.N_{0}=-154 \mathrm{dBm} / \mathrm{Hz}\right)$ is filtered into the considered signal band. The resulting synthetic received signal available at the observation time-stamp $t_{n}$ is thus given by:

$$
\begin{aligned}
W_{s}\left(t_{n}, \tau\right)= & \hat{h}\left(t_{n}, \tau\right) \otimes w_{0}(\tau, B)+n\left(t_{n}, \tau, B\right) \\
= & \sum_{j=1}^{\hat{L}_{p}\left(t_{n}\right)} \hat{\alpha}_{j}\left(t_{n}\right) w_{0}\left(\tau-\hat{\tau}_{j}\left(t_{n}\right), B\right) \\
& +n\left(t_{n}, \tau, B\right)
\end{aligned}
$$

where $w_{0}(\tau, B)$ is the reference template and $n\left(t_{n}, \tau, B\right)$ is the band-limited noise process at the observation time-stamp $t_{n}$ in the occupied band $B$.

For our simulation needs, in order to enable a dynamic variation of $S N R\left(t_{n}\right)$ and to preserve the natural relative power fluctuations due to body obstructions (as observed during the measurements campaign), we set and control the $S N R$ values a priori for an arbitrary reference time stamp (preferably in LOS). In our case, the reference time $t_{0}$ is for instance chosen when the received channel exhibits a maximum of the power transfer function $P(t)$. Imposing a priori the reference value $S N R\left(t_{0}\right)$ (as an input parameter) and given the actual $P\left(t_{n}\right)$ (and hence $P\left(t_{0}\right)$ ) directly available from measurements at any time-stamp $t_{n}$, the instantaneous $S N R\left(t_{n}\right)$ is then forced and scaled artificially so as to vary realistically over the entire acquisition duration, as follows:

$$
\left.S N R(t)\right|_{d B}=\left.S N R\left(t_{0}\right)\right|_{d B}+\left.P(t)\right|_{d B}-\left.P\left(t_{0}\right)\right|_{d B}
$$

where $S N R(t)$ is the re-scaled instantaneous signal energy to noise ratio, $S N R\left(t_{0}\right)$ and $P\left(t_{0}\right)$ are respectively the controlled $S N R$ value and power transfer function at time-stamp $t_{0}$, and $P(t)$ is the power transfer function at time $t$. In our study, $\operatorname{SNR}\left(t_{0}\right)$ is viewed as in imposed input parameter, which remains constant and valuable for the whole duration of one walk cycle, and over several noise process realizations (i.e. over which statistics are drawn). Practically, before applying (8) to account for the overall walk duration from the reference time stamp $t_{0}$, given the fixed filtered noise power imposed by $B$ and $N_{0}$, we re-scale the synthetic multipath impulse response $\hat{h}\left(t_{0}, \tau\right)$ in $(7)$ into $\hat{h}_{r}\left(t_{0}, \tau\right)$ so that $W_{s, r}\left(t_{0}, \tau\right)=\hat{h}_{r}\left(t_{0}, \tau\right) \otimes w_{0}(\tau, B)+n\left(t_{0}, \tau, B\right)$ can respect the input parameter $S N R\left(t_{0}\right)$ (and thus, applying the same scaling factor to the useful signal for each random noise process realization), as follows:

$$
\left.S N R\left(t_{0}\right)\right|_{l i n}=\frac{\int\left[W_{s, r}\left(t_{0}, \tau^{\prime}\right)-n\left(t_{0}, \tau^{\prime}, B\right)\right]^{2} d \tau^{\prime}}{N_{0}}
$$

The rationale for parameterizing the error model with $S N R\left(t_{0}\right)$ are twofold: i) we have noticed that the error regime is rather stable over LOS or NLOS portions of a given walk (i.e. exhibiting approximately the same statistics under relatively small variations of the instantaneous SNR) but mostly conditioned on body shadowing and ii) $S N R\left(t_{0}\right)$ shall be easier to predict once for all at the beginning of the walk cycle in localization-oriented simulations (e.g. with classical freespace propagation models) for being advantageously associated with LOS conditions. 

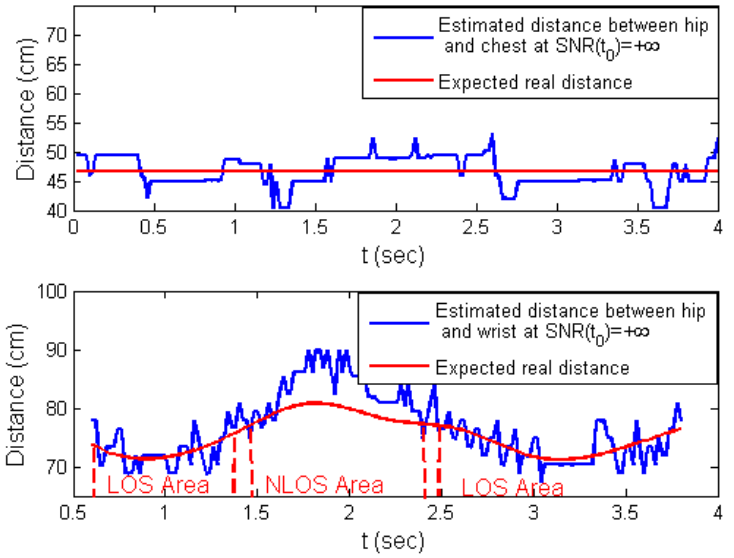

Figure 3: Equivalent inter-node distance retrieved out of correlation-based TOA estimation without noise (blue) and fitted reference distance after averaging with a sliding window and splines interpolation over the detected NLOS time stamp region (red), for both Hip-Chest (top) and Hip-Wrist links (bottom).

\subsection{Emulated TOA Estimates and Conditional Error Regimes}

At each observation time-stamp $t_{n}$, one TOA is thus estimated from each synthesized noisy received signal, using a matched filter as described in section 2.2 , i.e. by looking for the time shift that maximizes the cross-correlation function between the synthetic received signal $W_{s, r}\left(t_{n}, \tau\right)$ and the reference template $w_{0}(\tau, B)$, with an observation window of $5 n s$, like in [5], [10]. The window duration is sufficient to comply with an arrival time corresponding to the maximum distance between two synchronized nodes placed on the same body.

The first Hip-Chest link to be considered is always assumed in LOS conditions, whereas the Hip-Wrist link varies dynamically, leading periodically and alternatively to LOS and NLOS conditions. In order to classify the obstruction conditions, the retained method is based on the power transfer function. Relying on the initial measurements, the channel is considered in LOS (resp. NLOS) conditions whenever its power transfer function is larger (resp. lower) than $-60 \mathrm{~dB}$ (resp. $-65 \mathrm{~dB}$ ). The remaining unspecified time area is considered as a transition zone, with a steep power transition regime. Alternatively, the channel delay spread, which exhibits smaller values in LOS and higher values in NLOS conditions, could have been used to identify the channel obstruction configurations.

Finally, during the initial communication-oriented measurement campaign reported in [3], the real distance between nodes was not collected, since measurements were not carried out for localization purposes. However, in first approximation, one can try to extract this distance out of the measured TOA in time-stamp regions when the LOS conditions are clearly identified and with $S N R\left(t_{0}\right)=+\infty$ for the synthetic received signals in the largest bandwidth

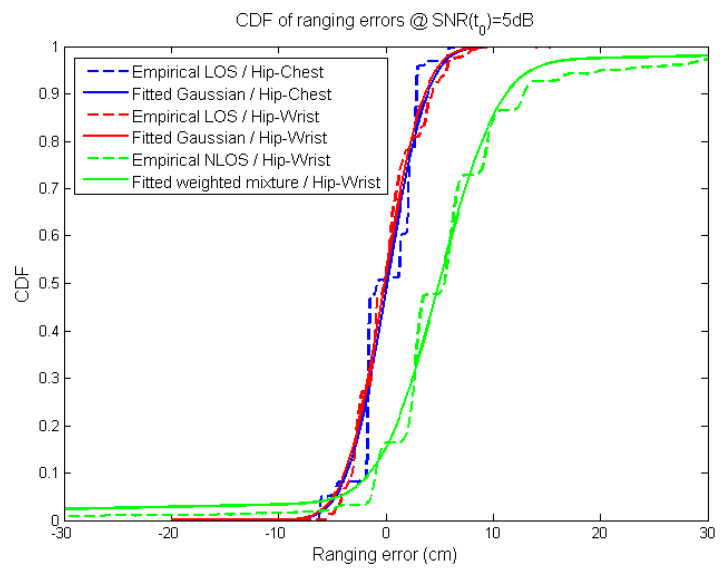

Figure 4: Empirical and model-based CDFs of ranging errors in both LOS and NLOS conditions, with $S N R\left(t_{0}\right)=5 d B$ in the band $[3.1,5.1] \mathbf{G H z}$.

$[3.1,5.1] \mathrm{GHz}$. Practically, the first Hip-Chest link is considered as fixed and the reference distance extraction was directly realized by averaging all the TOA measurements issued from MF estimation over the walk cycle to reduce TOA estimation errors appeared during the multipath extraction phase in the presence of overlapping components. Nevertheless, for the second Hip-Wrist link, a smoothing process was performed in a sliding window whose length corresponds to 20 consecutive time-stamp samples (e.g. within $20 \times 20 \mathrm{~ms}=400 \mathrm{~ms}$ ). The true distance was subsequently interpolated over NLOS areas, assuming continuity of the true distance at LOS/NLOS boundaries but discontinuity for the smoothed version of the measured distance (obtained with the sliding window). The idea consists in relying on the known extracted LOS portions, thus forming an time-stamp basis to infer the true distance in unknown NLOS timestamp areas through spline-based data extrapolation. Figure 3 intends to clarify the method used to determine the reference distance, assuming the latter will correspond to the so-called "expected real" distance while computing the ranging error in the following.

\section{RESULTS}

In this section we statistically characterize the obtained TOAbased ranging errors in the $[3.1,5.1] \mathrm{GHz}$ and $[3.75,4.25] \mathrm{GHz}$ frequency bands, for the two kinds of radio links. As previously mentioned, these models are conditioned on the channel obstruction status and on the reference $S N R\left(t_{0}\right)$. While running simulations, for each $S N R\left(t_{0}\right)$ value, 100 independent noise process realizations are drawn for the walk cycle duration. Over these realizations, for each frequency band, up to 20000 range measurements are then collected in LOS conditions for the Hip-Chest link, whereas 8600 and 3800 measurements are generated for the Hip-Wrist link, respectively in LOS and NLOS conditions.

\subsection{LOS Model}

Conditioned on the LOS case, it appears that the step-wise empirical Cumulative Density Function (CDF) of emulated range measurements enjoys a rather satisfactory fit (in a least squares sense) to the CDF of a Gaussian random vari- 


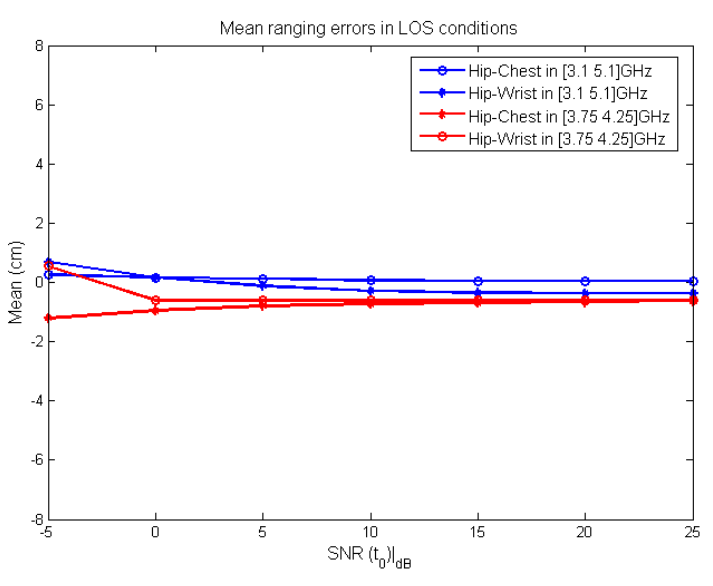

Figure 5: Mean of ranging errors in LOS conditions, as a function of $S N R\left(t_{0}\right)$.



Figure 6: Standard deviations of ranging errors $\sigma$ in LOS and NLOS conditions, as a function of $S N R\left(t_{0}\right)$.

able, whose standard deviation $\sigma$ is on the order of the time base period. Figure 4 shows examples for both simulationbased and model-based LOS CDFs with $S N R\left(t_{0}\right)=5 d B$ in the band $[3.1,5.1] \mathrm{GHz}$

Figures 5 and 6 show respectively the variations of the mean and standard deviation of the corresponding Gaussian LOS model for both links and both bands, as a function of $S N R\left(t_{0}\right)$. As seen in Figure 5, the mean varies around zero, with very low values (in comparison with the nominal expected true range value), and hence, it can be considered as null in first approximation over the explored range of $S N R\left(t_{0}\right)$ values. Figure 6 shows that the behavior of the standard deviation is asymptotically constant when $S N R\left(t_{0}\right)$ reaches a value of 10dB. At high SNRs, the strongest path detected through cross-correlation indeed coincides systematically with the direct path. The asymptotic error floor at high SNR thus depends mostly on the occupied band and center frequency, as discussed in [9].

To summarize, considering the tested Hip-Chest and HipWrist links, the distribution of the ranging error through

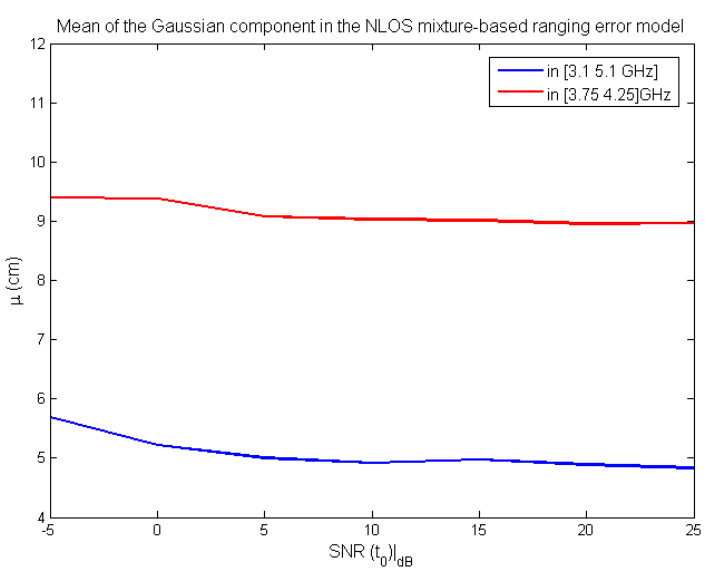

Figure 7: Mean value associated with the Gaussian part of the ranging error mixture-based model in NLOS conditions, as a function of $S N R\left(t_{0}\right)$.

correlation-based TOA estimation in LOS conditions in the $[3.1,5.1] \mathrm{GHz}$ and $[3.75,4.25] \mathrm{GHz}$ bands can be simply modeled as a centered Gaussian distribution, with a standard deviation depending on $B$ and $S N R\left(t_{0}\right)$ (See the legend of Figure 6 for detailed model parameters).

\subsection{NLOS Model}

In NLOS conditions (i.e. under body shadowing), the best fit has been obtained to a mixture-based model involving Gaussian and Uniform components. Figure 4 shows examples of both the empirical and model-based NLOS CDFs at $S N R\left(t_{0}\right)=5 d B$, in the $[3.1,5.1] \mathrm{GHz}$ band.

The corresponding conditional Probability Density Function $(\mathrm{PDF})$ is then expressed as follows:

$$
p(e)=\psi U\left(T_{w}\right)+(1-\psi) G\left(\mu, \sigma^{2}\right)
$$

where $p$ is the PDF of the ranging error $e$ in NLOS conditions, $U\left(T_{w}\right)$ is a uniform distribution, whose temporal support $T_{w}$ depends on the receiver observation window while performing TOA estimation through cross-correlation. Again, this window is chosen to enable detection within any intra-WBAN link after synchronization (e.g considering typically a worst case distance of $1.5 \mathrm{~m}), \psi$ is the weight of the uniform distribution, and $G\left(\mu, \sigma^{2}\right)$ is a Gaussian distribution with a mean $\mu$ and a variance $\sigma^{2}$.

The variation of those parameters in both bands of interest, as a function of $S N R\left(t_{0}\right)$ is represented in Figure 6, 7 and 8. As shown on Figure 8, at low $\operatorname{SNR}\left(t_{0}\right)$, the contribution of the uniform distribution component is high. This effect accounts for the distribution of the so-called apparent path arrival determined through cross-correlation over the entire observation window (e.g. between 0 and $5 n s$ ), when the noise level is so high that it can cause frequent missed detections or false alarms. The uniform weight in the mixture then directly reflects the probability of having either a false alarm or a missed detection. However, at higher $\operatorname{SNR}\left(t_{0}\right)$, the behavior is almost Gaussian, where the ranging error is centered around a positive mean, which can be interpreted as a positive bias caused by the obstruction of 


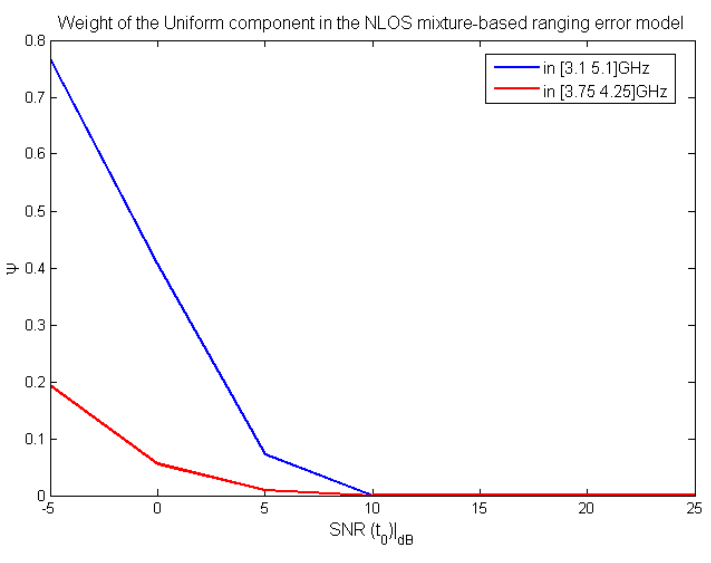

Figure 8: Weight of the Uniform part of the mixture-based ranging error model in NLOS conditions, as a function of $S N R\left(t_{0}\right)$.

the direct path (and hence, its apparent length extension). As shown in Figure 6, at high $S N R\left(t_{0}\right)$ (i.e. larger than $10 d B)$, in each operating band, the behavior of the error standard deviation in LOS is similar to the standard deviation of the Gaussian part of the mixture-based NLOS model, as the uniform weight is becoming quasi-null. Similar standard deviations means that the path detection performances are thus equivalently good in terms of dispersion in LOS and NLOS conditions, given the observed strongest path. However, it is worth keeping in mind that the apparent time of flight of the first observable path in NLOS cases is shifted independently of the path power, hence leading to a nonneglected ranging bias (i.e. besides random noise terms). The fact that the NLOS bias is approximately constant over $S N R\left(t_{0}\right)$ for a given band is also in line with the previous remarks. This very bias value, which seems to depend mostly on the occupied band, is rather hard to predict (as a deterministic parameter) and characterize further in practice. Hence, we recommend in our final ranging error model to assume this bias as a Uniformly distributed random variable, drawn once for all within a plausible range of a few tens of $\mathrm{cm}$ (i.e. approximately constant over all the NLOS portions of one given walk cycle).

\section{CONCLUSION}

In this paper we have characterized and modeled dynamic intra-WBAN ranging errors based on TOA estimation in the $[3.1,5.1] \mathrm{GHz}$ and $[3.75,4.25] \mathrm{GHz}$ frequency bands, the latter band being compliant with the mandatory band plan proposed in the IEEE 802.15.6 standard. The drawn models are based on preliminary intra-WBAN channel measurements performed in the band $[3.1,5.1] \mathrm{GHz}$, and conditioned on the channel configurations (LOS/NLOS). We have shown that the ranging error distribution could be modeled as a centered Gaussian distribution in LOS conditions, and as a weighted mixture between uniform and Gaussian distributions in NLOS conditions. In future works, this model shall be used to realistically assess the performance of new positioning and tracking algorithms, addressing the still challenging problem of large-scale individual motion capture based on stand-alone WBAN solutions.

\section{ACKNOWLEDGMENTS}

This work has been carried out in the frame of the CORMORAN project, which is funded by the French National Research Agency (ANR) under the contract number ANR11-INFR-010.

\section{REFERENCES}

[1] E. Ben Hamida, M. Maman, B. Denis, and L. Ouvry. Localization performance in wireless body sensor networks with beacon enabled mac and space-time dependent channel model. In Proceedings of IEEE PIMRC'10, the IEEE 21st International Symposium on Personal, Indoor and Mobile Radio Communications, (Istanbul, Sept. 26-29, 2010), pages 128-133, 2010.

[2] B. Denis and J. Keignart. Post-processing framework for enhanced uwb channel modeling from band-limited measurements. In Proceedings of IEEE UWBST'03, the IEEE Conference on Ultra Wideband Systems and Technologies (Reston, Sept. , 2003), 2007.

[3] R. D'Errico and L. Ouvry. Time-variant ban channel characterization. In Proceedings of IEEE PIMRC'09, the IEEE 20th International Symposium on Personal, Indoor and Mobile Radio Communications, (Tokyo, Sept. 13-16, 2009), pages 3000-3004, 2009.

[4] G. Destino, D. Macagnano, G. Abreu, B. Denis, and L. Ouvry. Localization and tracking for ldr-uwb systems. In Proceedings of IST Mobile Summit'07, the 16th IST Mobile and Wireless Communications Summit (Budapest, July 1-5, 2007), 2007.

[5] M. Di Renzo, R. Buehrer, and J. Torres. Ranging accuracy in uwb-based body area networks for full-body motion capture and gait analysis. In Proceedings of IEEE GLOBECOM'07, the 50th IEEE Global Telecommunications Conference (Washington DC, Nov. 26-30, 2007), 2007.

[6] S. Gezici, Z. Tian, G. Giannakis, H. Kobayashi, A. Molisch, H. Poor, and Z. Sahinoglu. Localization via ultra-wideband radios: A look at positioning aspects for future sensor networks. IEEE Signal Processing Magazine, 22(4):70-84, july 2005.

[7] K. Kwal, S. Ullah, and N. Ullah. An overview of ieee 802.15.6 standard. In Proceedings of ISABEL'10, the 3rd International Symposium Applied Sciences in Biomedical and Communication, (Rome, Nov. 7-10, 2010), 2010.

[8] Z. Mekonnen, E. Slottke, H. Luecken, C. Steiner, and A. Wittneben. Constrained maximum likelihood positioning for uwb based human motion tracking. In Proceedings of IPIN'10, the 1st International Conference on Indoor Positioning and Indoor Navigation, (Zurich, Sept. 15-17, 2010), pages 1-10, 2010.

[9] Z. Sahinoglu, S. Gezici, and I. Guvenc. Ultra-Wideband Positioning Systems: Theoretical Limits, Ranging Algorithms, and Protocols. Cambridge University Press, Cambridge, U.K., 2008.

[10] H. Shaban, M. El-Nasr, and R. Buehrer. Toward a highly accurate ambulatory system for clinical gait analysis via uwb radios. IEEE Transactions on Information Technology in Biomedicine, 14(2):284-291, march 2010. 